\title{
El rol de la mujer en la configuración del empresariado entre las décadas de 1950 y 1970
}

\author{
María E. Hernández ${ }^{1}$ \\ Universidad Pedagógica y Tecnológica de Colombia - UPTC \\ eliza794@yahoo.com \\ Patricia C. Barreto Bernal ${ }^{2}$ \\ Universidad Pedagógica y Tecnológica de Colombia - UPTC \\ patricia.barreto@uptc.edu.co \\ Óscar Gutiérrez Molina ${ }^{3}$ \\ Universidad Pedagógica y Tecnológica de Colombia - UPTC \\ oscar.gutierrezm@uptc.edu.co \\ DOI: https://doi.org/10.21158/01208160.n83.2017.1824
}

Fecha de recepción: 22 de febrero de 2017

Fecha de aprobación: 12 de junio de 2017

Cómo citar este artículo / To reference this article / Comment citer cet article / Para citar este artigo:

Hernández, M. E., Barreto, P. C. y Gutiérrez, O. (2017). El rol de la mujer en la configuración del empresariado entre las décadas de 1950 y 1970. Revista EAN, 83, pp 73 -92. https://doi.org/10.21158/01208160.n83.2017.1824

\section{Resumen}

Esta investigación buscó comprender el rol de la mujer en la configuración del empresariado boyacense entre las décadas de 1950 y 1970. Para tal fin, se consultaron las notarías primera y segunda de Tunja - Boyacá- que dieran fe de la constitución de empresas con participación femenina, y se realizaron entrevistas en profundidad a mujeres que crearon sus negocios en esta época e influyeron en el desarrollo empresarial de la región. Se concluyó del análisis documental y de la información obtenida de las entrevistas en profundidad, que el papel real y activo de la mujer en las décadas de 1950, 1960 y 1970 en los negocios de la región fue poco representativo, aun así, las mujeres participantes fueron líderes, aguerridas e irreverentes, que desafiaron los cánones sociales de la época con el fin de cumplir sus metas.

\section{Palabras clave}

Empresaria, mujer, género, empresas, rol.

1 Administradora de empresas por la Universidad Pedagógica y Tecnológica de Colombia; estudiante de maestría en Administración de Organizaciones. ORCID: http://orcid.org/0000-0002-6234-0989

${ }^{2}$ Administradora de empresas por la Universidad Pedagógica y Tecnológica de Colombia, magíster en Administración por el Instituto Tecnológico y de Estudios Superiores de Monterrey, y doctora en Administración por la Universidad Eafit. Docente de la Universidad Pedagógica y Tecnológica de Colombia. ORCID: http://orcid.org/0000-0001-5349-9712

${ }^{3}$ Administrador de empresas por la Universidad Nacional de Colombia, magíster en Administración por la Escuela Superior de Administración de Empresas, y doctor en Nuevas Tendencias en Dirección de Empresas por la Universidad de Salamanca. 


\title{
The role of women in the consolidation of entreprenuerhip between 1950 and 1970
}

\begin{abstract}
This research paper describes the role of women in the consolidation of entrepreneurship in Boyaca between 1950 and 1970. For this reason, the First and the Second Notaries in Tunja, Boyaca, were consulted to attest the foundation of companies managed by women, also interviewing those women who started their own companies during those decades and therefore impacted entrepreneurial development in this region. An analysis of documents and information resulting from those interviews concluded that the real and active role of women in business in the 50's, 60's and 70's was slightly representative, yet women participated as strong leaders who avoided social conventions to reach their goals.
\end{abstract}

Key words. Woman manager, woman, gender, companies, role.

\section{Le rôle des femmes dans la configuration de l'entreprenariat entre les années 1950 et 1970}

Résumé. Cette étude tente de montrer le rôle occupé par les femmes dans la configuration de l'entreprenariat de la région de Boyacá -Colombie - entre les années 1950 et 1970. Différents notaires de la ville de Tunja (Boyacá) ont été consultés donnant foi de la constitution d'entreprises de participation féminine. Plusieurs entretiens ont été réalisés avec des entrepreneuses ayant créé leur affaire à cette époque et qui ont influencé le développement entrepreneurial de la région. L'analyse documentaire et les informations recueillies montrent que le rôle réel des femmes entrepreneuses des années 1950 à 1970 de la région de Boyacá était peu représentatif dans l'économie locale bien que ces entrepreneuses soient des leaders aguerries défiant les normes sociales de l'époque pour atteindre leurs objectifs.

Mots clefs. Entrepreneuse, femme, genre, entreprises, rôle.

\section{O papel das mulheres na configuração do empresariado entre as décadas de 1950 e 1970}

Resumo. Esta pesquisa buscou compreender o papel das mulheres na configuração do empresariado de Boyacá -Colômbia - entre os anos 1950 e 1970. Para tanto, foram consultados os primeiro e segundo cartórios de Tunja, capital de Boyacá, para atestar a constituição de empresas com participação feminina, e se realizaram entrevistas em profundidade com mulheres que criaram seus negócios neste momento e influenciaram o desenvolvimento empresarial da região. A partir da análise documental e da informação obtida das entrevistas em profundidade concluiu-se que ainda que o papel real e ativo das mulheres nas décadas de 1950, 1960 e 1970 nos negócios da região tenha sido pouco representativo, as mulheres participantes foram líderes, aguerridas, e irreverentes e que desafiaram os cânones sociais da época com o fim de atingir seus objetivos.

Palavras-chave. Empresaria, mulher, gênero, empresas, papel. 


\section{Introducción}

T a finalidad de este estudio es comprender Lel rol de la mujer y su aporte a la configuración empresarial del departamento de Boyacá. Una de las razones por las cuales se realiza esta investigación es que existe un vacío con relación al papel que la mujer ha tenido en el desarrollo del empresariado boyacense. Aunque múltiples estudios han analizado el desarrollo empresarial y el empresariado en Colombia y Boyacá, ellos han sido generales sin distinción de género.

El artículo se ocupa del rol de la mujer en la configuración del empresariado boyacense desde la década de 1950 hasta la década de 1970, a través de cinco categorías o ejes temáticos; i) la participación económica en la empresa; ii) el papel de la mujer en la empresa; iii) la actividad económica ejercida en la empresa; iv) el tipo de figura jurídica social elegida; y v) la duración de la empresa en el mercado.

Después de realizado el análisis de la información proveniente de las fuentes primarias y secundarias, se encontró que el rol de la mujer en la configuración del empresariado boyacense de la década de 1950 hasta la década de 1970 no fue altamente significativo, pero sí creciente debido al impacto de los diversos factores políticos, sociales, económicos o culturales que caracterizaron el desarrollo y contexto general del país en esas décadas.

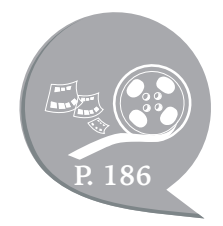

\section{Referentes conceptuales y teóricos}

os conceptos teóricos fundamentales con la historia de las empresas y sus actores, para luego centrarse en los referentes acerca del rol de género, de tal manera que se pueda comprender mejor cuál fue el abordaje conceptual que guió el desarrollo de la investigación y que sirvió como referente para interpretar los resultados.

\subsection{Historia empresarial}

En lo que concierne a la historia de las empresas, se debe precisar que está marcada por la lucha, debido a que existen «diversos agentes que participan en este campo, entre ellos los empresarios, los trabajadores, los sindicatos, la competencia, y sobre todo el Estado y los consumidores (clientes/ ciudadanos)» (Ortiz, Morales y León, 2014, p. 166). De esta forma, es un entramado de relaciones sociales entre diversos agentes.

Frente al tema, la Oficina Internacional del Trabajo (2007, p. 45) afirma que «con independencia de su tamaño o sector de operación, el contexto político, social, económico y cultural reviste importancia e impacto en la evolución histórica de todas las empresası. Lo cual implica que las empresas y sus actores son producto de la historia general de un país y una región, que en este caso es Colombia, y específicamente, Boyacá. 
Desde la perspectiva de la historia empresarial, Dávila (2012, pp. 64-65) propone diferentes unidades de análisis, a saber:

Empresas específicas (privadas, estatales y mixtas), empresarios (individuos) en diferentes sectores económicos (industria manufacturera, comercio, ganadería, minería, etc.), familias empresariales, grupos económicos, asociaciones de empresarios e instituciones para la educación de empresarios y de sus cuadros directivos (pp. 64-65).

En el caso particular de este estudio, la unidad de análisis son empresarios en una perspectiva societal, es decir, que se basa en la teoría social.

\subsection{Referentes sobre los estudios de género en el desarrollo empresarial}

Teniendo en cuenta que el desarrollo de la empresa y de sus actores es producto de un entorno social, ambiental, cultural, político y económico que ha caracterizado la historia general de los pueblos y las naciones, se precisa un análisis desde la perspectiva de género, tal como se propone en este trabajo de investigación, donde se analiza el rol de la mujer en las décadas de 1950 a 1970. Cuando se hace referencia al estudio de género, se está hablando directamente del papel que desempeñan las personas dependiendo de su sexo. Se hace mención de este tema debido a que el objetivo principal consiste en determinar cuál ha sido el rol de las mujeres en el desarrollo del empresariado. De acuerdo con el contexto social, se propone este enfoque sustentado en la perspectiva de Lamas (2003), citado por Macho y Rosales (2011) en el cual se debe «incorporar la categoría de género, ya que la visión sobre la explicación de la realidad social desde el pensamiento moderno no tiene en cuenta a los individuos concretos y diferenciados por sexo y raza». En otras palabras, las investigaciones anteriores no han considerado el género, de esta forma, no se sabe cómo o cuándo la mujer empezó a ser un actor activo del empresariado.

Para comprender el contexto de este análisis, se acude al concepto de género desde el enfoque social de Serret (2008, p. 15), que parte de:

[...] un punto de vista, en el cual se visualizan los distintos fenómenos de la realidad (científica, académica, social o política), que tiene en cuenta las implicaciones y efectos de las relaciones sociales de poder entre los géneros (masculino y femenino, en un nivel, y hombres y mujeres en otro).

En lo que concierne directamente al rol del género femenino como parte integral y activa del campo empresarial en Colombia $y$ el mundo, se enmarca en cada momento social e histórico en un contexto global, es decir, sujeto a las influencias y consecuencias de diferentes sucesos, tales como las guerras, los cambios políticos que le dieron mayor participación, los descubrimientos científicos que facilitaron su desarrollo, entre otros que le fueron abriendo campo en la actividad política y económica.

Como resultado de transformaciones en las condiciones económicas, políticas y sociales del mundo y el país, en el siglo XX, se presentó un despertar de la mujer que se hizo partícipe activa y gestora en diversos entornos distintos de su hogar. Como consecuencia de las luchas de los movimientos feministas, ula mujer empezó a tener una mayor participación en la esfera pública, logrando una incursión en el ambiente laboral, como sujeto activo en diferentes organizaciones de distintas naturalezası (Garbiras, 2010, p. 5). En una encuesta realizada por Gallup Colombia para la revista Semana (1995), la mayoría de los entrevistados admitía que las mujeres son más honestas y 
más eficientes en su trabajo que los hombres, tienen más sensibilidad social, son más responsables en el manejo de sus asuntos y tienen mayor capacidad para resolver conflictos.

Según Segura y Pintor (2010), el terreno que han venido ganando las mujeres colombianas se debe a que estas se caracterizan por ser emprendedoras, trabajadoras y dinámicas, con un alto sentido de la responsabilidad y muy perseverantes, son muy dedicadas en sus labores, sin descuidar su hogar y familia, con una suspicacia muy desarrollada así como un carácter fuerte y temperamental, pujantes, suelen conquistar sus objetivos, inclusive, en los entornos más adversos, lo cual las hace altamente efectivas.
Aunque en las últimas décadas el ingreso de las mujeres al mundo laboral ha tenido un crecimiento acelerado, su ascenso en la escala corporativa ha mostrado ser más lento. De acuerdo con los datos existentes en el contexto global, la presidencia de las compañías y la alta gerencia sigue estando a cargo principalmente de varones, situación evidentemente inequitativa si se consideran las actuales condiciones de las mujeres en el ámbito educativo y social. Las razones de este fenómeno aún no son claras y no se tiene una explicación que tenga relación con las capacidades vinculadas al sexo. Así, el asunto parece estar más relacionado con variables psicológicas, sociales y culturales que deben ser estudiadas a fondo (Contreras, Pedraza y Mejía, 2012, p. 184).

\section{Metodología}

Según Dávila (2012, p. 35), la historia Oempresarial se nutre de la información y los datos que proceden de los distintos archivos y de la teoría que permite analizar esta documentación histórica. Naturalmente, una primera operación del investigador consiste en la descripción ordenada de la historia de la empresa o del empresario que estudie, pero se trata de un primer paso que hay que superar si se quiere comprender el comportamiento de la empresa y su éxito o fracaso. Y para explicar hay que recurrir a la utilización de la teoría de la empresa, que permite interpretar los datos históricos. Esta investigación sigue un enfoque cualitativo en cuanto se interesa por develar el papel de las mujeres en un rol socioeconómico innovador y retador para la mitad del siglo XX en un departamento históricamente conservador.
Como estrategia de investigación, se acudió al análisis documental a partir del uso de diferentes tipos de documentos sistemáticamente obtenidos a través de fuentes archivísticas, como los registros de las notarías primera y segunda y de la Cámara de Comercio de Tunja, prensa regional y local. La información se clasificó en categorías de análisis a través de una matriz, la cual sirvió de base para recoger y sistematizar la información recopilada. También se utilizaron como estrategia complementaria las historias de vida a través de entrevistas en profundidad, con el fin de recopilar de fuente primaria las experiencias y percepciones de seis mujeres empresarias boyacenses que fueron económicamente activas en el periodo de estudio y cuyos nombres emergieron de la revisión exhaustiva de los archivos 
notariales El análisis buscó identificar las cualidades empresariales de las mujeres y conocer su papel en aquella época. Las entrevistas en profundidad se realizaron conforme a los parámetros del Esquema Analítico para Adelantar Estudios de Historia Empresarial (EAHE) de Carlos Dávila.
Además de la información primaria proveniente de los archivos de las notarías primera y segunda de Tunja y de las entrevistas a las empresarias de las décadas de 1950, 1960 y 1970 , se revisaron fuentes secundarias, como artículos que sirvieron de complemento de la información y hallazgos obtenidos.

\section{Resultados y discusión}

$\mathrm{T}$ eniendo en cuenta que «las organizaciones existen en un lugar y en un momento histórico determinado, estas tienden a integrar los elementos de su propio contexto social y a reproducir exponencialmente las características de lo que se considera 'femenino' o 'masculino'» (Zabludovsky, 2015, p. 73). Para este análisis, se toma como precedente los objetivos propuestos y los referentes teóricos que dan una visión del papel de la mujer no solo en el ámbito empresarial sino en el social, lo cual implica un análisis de resultados provisto de argumentaciones que los ratifiquen y contextualicen.

La dimensión temporal resulta en particular importante en una investigación que se ubica teóricamente en el campo de la historia empresarial, no solo porque se acota al tiempo en que ocurrieron acontecimientos sociales que determinaron cambios relevantes para el papel de la mujer en la sociedad, sino por los procesos de transformación social y cultural que se dieron como parte de la modernización de una región y de un país. A continuación, se presentan los resultados más significativos en orden temporal para dar luego paso a los hallazgos respeto de la importancia del género en la conformación de la élite empresarial boyacense.

\subsection{Mujeres que han creado empresas en el departamento de Boyacá en las décadas de 1950 hasta la década de 1970}

Las décadas de 1950, 1960 y 1970 fueron testigo de importantes transformaciones en la manera en que la mujer participó en la modernización del país y en el desarrollo de nuevas prácticas, laborales y culturales que replantearon la organización de las actividades del trabajo y del hogar. Al respecto, Reyes (2015) afirma que, durante la segunda mitad del siglo XIX y la primera mitad del siglo XX, se consideró a la mujer dentro de los cánones sociales y religiosos como reina del hogar, exigiendo que las actividades que realizaran fueran las concernientes al cuidado de su esposo y sus hijos asumiendo la responsabilidad de la educación y la evangelización de la religión católica. El papel asignado por la sociedad burguesa limitó a las mujeres al 
rol de misioneras sociales, encargándolas de moralizar a las mujeres y a los niños de los sectores marginados, situación que permitió a las mujeres de élite trascender el espacio de amas de casa y obtener un lugar destacado en la sociedad.

Según Londoño (1995), en Colombia, las mujeres tuvieron que esperar hasta la década de 1930 para que sus derechos políticos empezaran a ser reconocidos, $\mathrm{y}$ hasta mediados del siglo para poder votar, siendo el país uno de los últimos en Occidente en reconocer el sufragio femenino. Durante el resto del siglo, las mujeres tomaron parte activa en las guerras civiles y en los principales acontecimientos políticos; esta participación estuvo limitada a la posición social económica y política de las mujeres, es decir, las mujeres que tuvieron influencia histórica en cualquier campo estaban relacionadas con hombres que tenían influencia en el país.

A pesar del limitado papel en lo social y económico que tuvieron las mujeres en las décadas de 1950, 1960 y 1970, el estudio del rol de la mujer en la configuración del empresariado boyacense durante estas tres décadas - realizado a través de 158 registros notariales-, evidencia una intervención activa en este proceso, inicialmente restringida a la participación económica como propietaria por herencia o por lazos familiares y posteriormente hacia el final del periodo estudiado como líderes activas con roles directivos y gerenciales.

\subsection{Participación económica de la mujer en la creación de empresas en Boyacá}

En lo que respecta a la participación de las mujeres como accionistas o aportantes económicas en la creación o configuración de las organizaciones empresariales en Boyacá, la investigación muestra que aproximadamente el aporte de capital de cada mujer es de $25 \%$ para la década de 1950 , $37 \%$ para la década de 1960 , y $34 \%$ para la década de 1970, para una participación promedio general en estos treinta años de 33 $\%$ (Tabla 1). Esto indica, que en este periodo, las mujeres aparecían en el empresariado boyacense como accionistas minoritarias, lo cual en consecuencia y de acuerdo con los principios de la gestión administrativa, no les daría mayor influencia en la toma de decisiones. Además, según entrevistas, este porcentaje era el resultado de un contexto social en el cual la mujer desempeñaba un papel pasivo debido a que eran consideradas como amas de casa que debían responder por la administración del hogar y el cuidado de los hijos. En este estudio se evidencia que la mujer empieza a desligarse de ese concepto y comienza a tener independencia en el manejo de sus propios recursos.

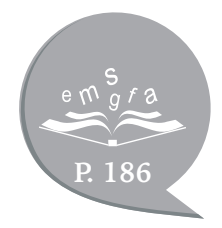


Tabla 1. Participación económica de la mujer en la creación de la empresa boyacense (1950-1979).

\begin{tabular}{|c|c|c|c|c|c|c|c|c|}
\hline \multirow{3}{*}{$\begin{array}{c}\text { Rango } \\
\text { accionario (\%) }\end{array}$} & \multicolumn{2}{|c|}{$1950-1959$} & \multicolumn{2}{|c|}{$1960-1969$} & \multicolumn{2}{|c|}{$1970-1979$} & \multicolumn{2}{|c|}{ Total } \\
\hline & \multicolumn{2}{|c|}{ Mujeres } & \multicolumn{2}{|c|}{ Mujeres } & \multicolumn{2}{|c|}{ Mujeres } & \multicolumn{2}{|c|}{ Mujeres } \\
\hline & Cantidad & $\%$ & Cantidad & $\%$ & Cantidad & $\%$ & Cantidad & $\%$ \\
\hline $0.1-10$ & 4 & 25 & 2 & 8 & 12 & 9 & 18 & 10 \\
\hline $10.1-20$ & 2 & 13 & 2 & 8 & 25 & 18 & 29 & 16 \\
\hline $20.1-30$ & 5 & 31 & 2 & 8 & 10 & 7 & 17 & 9 \\
\hline $30.1-40$ & 1 & 6 & 3 & 12 & 15 & 11 & 19 & 11 \\
\hline $40.1-50$ & 3 & 19 & 15 & 60 & 71 & 51 & 89 & 50 \\
\hline Más del 50 & 1 & 6 & 1 & 4 & 5 & 4 & 7 & 4 \\
\hline Total & 16 & 100 & 25 & 100 & 138 & 100 & 179 & 100 \\
\hline Promedio aporte & & 25 & & 37 & & 34 & 18 & 10 \\
\hline
\end{tabular}

Fuente. Elaboración propia.

\subsubsection{Papel de la mujer en la dirección de la empresa boyacense}

El estudio de 158 empresas muestra que de 1950 a 1979 solo $15 \%$ de estas contaba con la participación activa de una mujer en el cargo de gerente o director; sin embargo, se resalta el hecho de que

la participación femenina en el liderazgo empresarial para este periodo tuvo una tendencia creciente, es decir, se pasó de 1 mujer en la década de 1950 a 19 mujeres en la década de 1970 (Tabla 2).

Tabla 2. Papel de la mujer en la dirección de la empresa boyacense (1950-1979).

\begin{tabular}{|l|c|c|c|c|c|c|c|c|}
\hline \multirow{2}{*}{} & \multicolumn{2}{|c|}{$1950-1959$} & \multicolumn{2}{c|}{$1960-1969$} & \multicolumn{2}{c|}{$1970-1979$} & \multicolumn{2}{c|}{ Total } \\
\cline { 2 - 9 } & \multicolumn{2}{|c|}{ Gerentes } & \multicolumn{2}{c|}{ Gerentes } & \multicolumn{2}{c|}{ Gerentes } & \multicolumn{2}{c|}{ Gerentes } \\
\cline { 2 - 9 } & Cantidad & $\%$ & Cantidad & $\%$ & Cantidad & $\%$ & Cantidad & $\%$ \\
\hline Mujer gerente & 1 & 7 & 3 & 10 & 19 & $\underline{17 \%}$ & $\underline{\mathbf{2 3}}$ & $\underline{15 \%}$ \\
\hline Hombre gerente & 11 & 79 & 20 & 65 & 69 & $61 \%$ & 100 & $63 \%$ \\
\hline $\begin{array}{l}\text { No registra quién } \\
\text { gerencia }\end{array}$ & 2 & 14 & 8 & 26 & 25 & $22 \%$ & 35 & $22 \%$ \\
\hline Total & 14 & 100 & 31 & 100 & 113 & $100 \%$ & 158 & $100 \%$ \\
\hline
\end{tabular}

Fuente. Elaboración propia. 
Estos resultados son coherentes con el contexto social, económico, cultural y político del país en la época. A inicios de la década de 1950 se enaltecía su labor como esposa y madre, pero:

[...] "después de 1957 con el otorgamiento de la ciudadanía y los cambios sociodemográficos, como el acceso a la educación, la planificación familiar, y la reducción del número de hijos, la mujer experimenta una mayor autonomía económica y social, que le abre la puerta al ingreso laboral y empresarial (Londoño y Gómez, 2010, pp. 333-334).

Todos estos cambios se reflejan en un crecimiento del liderazgo de la mujer que se materializa en la dirección de empresas hacia finales de 1970. «Esta realidad comienza a crear cierta conciencia sobre el rol de las mujeres en puestos directivos propios $y$ liderazgo, donde ella materializa modelos y formas de gestión hasta ahora invisibles, inhabituales y no reconocidos» (Rodríguez, Jiménez y Rebollo, 2016, p. 345).

De esta forma, los datos encontrados muestran, que si bien todavía existía desigualdad debido al contexto sociopolítico de la época, el rol de la mujer estaba cambiando desde el punto de vista empresarial, es decir, se estaba dando una mayor inmersión de estas en las empresas o bien como trabajadoras o bien en el papel de directivas, denotándose claramente que su papel de madre y esposa podía integrarse a sus actividades productivas o anteponerse al cumplimiento del ideal de una familia. Frente al tema, Londoño y Gómez (2010) asienten que para esta época, en lo que se refiere al mundo laboral y empresarial, las mujeres tienen una acelerada vinculación, sobre todo, en edades entre los 25 y 29 años, lo cual también fue causa y fortalecimiento de cambios en las constituciones familiares, es decir, aparecen las uniones libres, los hogares uniparentales, la mujeres viudas a causa de la violencia, entre otros factores que las obligaron a trabajar y a crear unidades económicas para poder mantener a sus familias.

\subsubsection{Actividad económica de la mujer empresaria}

Otro de los aspectos que se identifican en este estudio es el tipo o clase de actividad a las que se dedicaban las empresas que tenían participación femenina, para lo cual se tuvieron en cuenta cuatro sectores básicos: i) comercio, ii) servicios, iii) industria y iv) explotación primaria.

En la década de 1950, las actividades empresariales con mayor auge y acogida por las mujeres empresarias de Boyacá estaban relacionadas con los sectores de comercio, industria y explotación primaria, referidas específicamente al comercio de ropa y otros enseres, y la explotación y trasformación de productos agropecuarios (Tabla 3). El desarrollo de estas actividades empresariales es consecuente con los conocimientos previos que las mujeres tenían al ejercer su papel como madres y esposas, es decir, conocían las necesidades y los requerimientos del comercio de los productos de consumo masivo, como ropa, comida, electrodomésticos y enseres; así mismo, sabían de la producción agropecuaria, pues estaban vinculadas a las prácticas propias del hogar.

En la década de 1960, se evidenció una mayor participación del comercio, industria y servicios; en el caso del comercio, su participación fue de $35 \%$, sobresaliendo actividades como la compraventa de mercancías para el hogar y productos de consumo masivo y abarrotes; en el caso de los servicios, estos se centraron en la venta de seguros y títulos empresariales (Tabla 3). Estos resultados muestran una relación directa con la situación económica del país para ese momento, es decir, tal como lo afirma Garay (1998), uen 
esta época, la demanda del mercado doméstico absorbía la casi totalidad de la producción industrial del paísy. Esto significa que la producción estaba centrada en la satisfacción de las familias colombianas, lo cual llevaba al incremento del comercio de productos para el hogar.

En la década de 1970, sobresale notoriamente el sector servicios con participación de $50 \%$ seguido del comercio con $29 \%$ (Tabla 3). En el primer caso, se encontraron actividades relacionadas con la venta de paquetes turísticos, los seguros, los servicios empresariales de gestión y administración, el trasporte de todo tipo, entre otros. El comercio, entretanto, se centró, básicamente, en actividades de compraventa de abarrotes y productos para el hogar.

El incremento de la actividad empresarial en el sector servicios del empresariado femenino boyacense en la década de 1970 (Tabla 3) es congruente con la evolución de los diferentes sectores económicos en Colombia y el mundo, pues, según Bernal, Cañas, Castillo, Cortés, Corredor y Quintero (2011), el aumento del sector servicios en el Producto Interno Bruto (PIB) desde la década de 1970 ha sido un común denominador, resultado natural del proceso de desindustrialización. Esta afirmación la ratifica Maldonado (2010, pp. 33-34): «A partir de 1970 hasta final del siglo es evidente la desaceleración en los ritmos de crecimiento del sector agropecuario e industrial dentro de la estructura productiva nacional». Esto es reflejo del proceso de reestructuración regresiva de la producción iniciado por la economía colombiana, especialmente desde principios de la década de 1970, cuando el sector primario comienza a perder participación de manera importante y sostenida, mostrando históricamente una notable reducción en su participación en el PIB total.

Tabla 3. Actividad económica de la empresa con participación de la mujer boyacense (1950-1979).

\begin{tabular}{|l|c|c|c|c|c|c|c|c|}
\hline \multirow{2}{*}{} & \multicolumn{2}{|c|}{$1950-1959$} & \multicolumn{2}{c|}{$1960-1969$} & \multicolumn{2}{c|}{$1970-1979$} & \multicolumn{2}{c|}{ Total } \\
\cline { 2 - 10 } & \multicolumn{2}{|c|}{ Mujeres } & \multicolumn{2}{c|}{ Mujeres } & \multicolumn{2}{c|}{ Mujeres } & \multicolumn{2}{c|}{ Mujeres } \\
\cline { 2 - 10 } & Cantidad & $\%$ & Cantidad & $\%$ & Cantidad & $\%$ & Cantidad & $\%$ \\
\hline Comercio & 4 & 29 & 11 & 35 & 33 & 29 & 48 & 30 \\
\hline Servicios & 0 & 0 & 7 & 23 & 56 & 50 & 63 & 40 \\
\hline Industria & 5 & 36 & 6 & 19 & 13 & 12 & 24 & 15 \\
\hline $\begin{array}{l}\text { Explotación } \\
\text { primaria }\end{array}$ & 5 & 36 & 2 & 6 & 9 & 8 & 16 & 10 \\
\hline No registra & 0 & 0 & 5 & 16 & 2 & 2 & 7 & 4 \\
\hline Total & 14 & 100 & 31 & 100 & 113 & 100 & 158 & 100 \\
\hline
\end{tabular}

Fuente. Elaboración propia. 


\subsubsection{Tipo de figura jurídica societaria de las empresas creadas}

Con relación a la figura jurídica que crearon las mujeres boyacenses de las décadas de 1950 a 1970, sobresalen significativamente las sociedades limitadas ${ }^{4}$ que en términos generales durante este periodo tienen una participación de $89 \%$. Las razones por las que las empresarias boyacenses constituyeron este tipo de figura jurídica están relacionadas directamente con la novedad que esta clase de organización represen- taba, además de las ventajas comerciales que para el momento ofrecían, las cuales se relacionan con el capital mínimo de constitución, la participación directa en beneficios, la facilidad de administración, la posibilidad de reunir un mayor capital para la empresa, entre otras. Esto quiere decir, que las mujeres podrían ser accionistas pero no necesariamente tendrían que ser partícipes de la dirección y el control de ellas, además, su responsabilidad se limitaría al porcentaje que aportaron (Tabla 4).

Tabla 4. Tipo de sociedad constituida por las mujeres boyacenses (1950-1979).

\begin{tabular}{|l|c|c|c|c|c|}
\hline \multirow{2}{*}{\multicolumn{1}{c|}{ Tipo de sociedad }} & $1950-1959$ & $1960-1969$ & $1970-1979$ & \multicolumn{2}{c|}{ Total } \\
\cline { 2 - 6 } & $\begin{array}{c}\text { Cantidad } \\
\text { sociedades }\end{array}$ & $\begin{array}{c}\text { Cantidad } \\
\text { sociedades }\end{array}$ & $\begin{array}{c}\text { Cantidad } \\
\text { sociedades }\end{array}$ & $\begin{array}{c}\text { Cantidad } \\
\text { sociedades }\end{array}$ & $\%$ \\
\hline Sociedad limitada & 13 & 30 & 98 & 141 & 89 \\
\hline Sociedad en comandita & 0 & 0 & 10 & 10 & 6 \\
\hline Otras (empresas unipersonales) & 1 & 1 & 5 & 7 & 4 \\
\hline Total & 14 & 31 & 113 & 158 & 100 \\
\hline
\end{tabular}

Fuente. Elaboración propia.

\subsubsection{Expectativa de duración de las empresas conformadas por mujeres (1950-1979)}

Para el caso, se encontró que la mayor tendencia está en la proyección definida para un periodo de duración entre nueve y diez años, debido a que este periodo cuenta con 29,32 y $25 \%$ para las décadas de 1950, 1960 y 1970. Así mismo, se halló un incremento en la confianza, el cual se evidencia en el hecho de que para la década de 1970 crece la participación hasta $42 \%$; estos hechos muestran que en cuestiones de negocios las empresas que integraban mujeres tenían una expectativa a largo plazo, es decir, se proyectaban en principios de optimismo, decisión y trascendencias, en busca de aportar y figurar en el desarrollo empresarial de la región.

Tomando como referente los promedios de tiempo estipulado para las empresas en el momento de la constitución por décadas,

\footnotetext{
${ }^{4}$ La Sociedad de Responsabilidad Limitada «cuenta con un número limitado de accionistas (entre 2 y 25 dueños) y sus acciones no son negociables. Es una figura doblemente limitada: tanto limitada en sus obligaciones legales como en el número de sus dueños») (Hernández, 2005, p. 140).
} 
se encontró que para la década de 1950 es de 5.7 años, para la de 1960 también de 5.7 años y para la de 1970 aumenta a los 7.5 años, con un promedio total de siete años. A pesar de los periodos definidos muchas de estas empresas han trascendido y se han convertido en íconos del desarrollo empresarial y económico de la región. Según entrevistas, esto es el resultado de hechos que muestran mayor liderazgo, seguridad, confianza y autonomía en las decisiones (Tabla 5).

Tabla 5. Duración de la empresa en el mercado (1950-1979).

\begin{tabular}{|l|c|c|c|c|c|c|c|c|}
\hline & \multicolumn{2}{|c|}{$1950-1959$} & \multicolumn{2}{c|}{$1960-1969$} & \multicolumn{2}{c|}{$1970-1979$} & \multicolumn{2}{c|}{ Total } \\
\cline { 2 - 10 } & \multicolumn{2}{|c|}{ Empresas } & \multicolumn{2}{c|}{ Empresas } & \multicolumn{2}{c|}{ Empresas } & \multicolumn{2}{c|}{ Empresas } \\
\cline { 2 - 10 } & Cantidad & $\%$ & Cantidad & $\%$ & Cantidad & $\%$ & Cantidad & $\%$ \\
\hline 0-2 años & 2 & 14 & 1 & 3 & 2 & 2 & 5 & 3 \\
\hline 3-4 años & 2 & 14 & 3 & 10 & 2 & 2 & 7 & 4 \\
\hline 5-6 años & 3 & 21 & 6 & 19 & 18 & 16 & 27 & $17 \%$ \\
\hline 7-8 años & 1 & 7 & 1 & 3 & 1 & 1 & 3 & 2 \\
\hline 9-10 años & 4 & 29 & 10 & 32 & 28 & 25 & 42 & $27 \%$ \\
\hline $\begin{array}{l}\text { Más de 10 } \\
\text { años }\end{array}$ & 1 & 7 & 3 & 10 & 47 & 42 & 51 & $32 \%$ \\
\hline No registra & 1 & 7 & 7 & 23 & 15 & 13 & 23 & $15 \%$ \\
\hline Total & 14 & 100 & 31 & 100 & 113 & 100 & 158 & $100 \%$ \\
\hline Promedio & & 5.79 & & 5.71 & & 7.54 & & 7.02 \\
\hline
\end{tabular}

Fuente. Elaboración propia.

La trascendencia empresarial también es consecuencia de los cambios y la influencia de países en vías de desarrollo. Así, de acuerdo con Boserup (1970), citado por Moghadam (1999), «el surgimiento de la mujer asalariada que empezó en la década de 1970, seguido de un periodo temprano de desarrollo capitalista y crecimiento económico, caracterizado por el desplazamiento del trabajo artesanal, comercialización de la agricultura y migración rural a urbana» (p. 372), llevó a un fortalecimiento empresarial en todos los sectores dando mayor posibilidad al género femenino.

La poca duración de las empresas creadas por empresarias boyacenses en las décadas de 1950 a 1970 es producto de la situación socioeconómica del país, pues las dificul- tades del sector empresarial comenzaron con la caída de los precios del café hacia comienzos de 1955, momento en el que se inicia una de las crisis más profundas que ha vivido la economía colombiana y que tendría como consecuencia la caída de Rojas Pinilla en 1957. En un primer momento, el desgastado gobierno militar no enfrentó la situación y continúo con su alto ritmo de gastos hasta llevar la economía al borde del colapso (Díaz, 1972). Esta situación condujo a que muchas empresas, sin importar su tamaño, tuvieran que dejar de operar, pues no se tenía rentabilidad sino déficit. Además de los factores políticos que indudablemente afectaron el desarrollo de las empresas, están los factores económicos. En este caso, se encontró que, ua principios de los setentas, la inflación aumentó sorpresivamente, por 
una fase expansiva del ciclo de la actividad económica que aumentó la inflación de productos importados y un choque de oferta en la agricultura de alimentos, junto con una fuerte expansión del gasto público» (Kalmanovitz y López, s. f., p. 33). Así, la volatilidad del índice de precios al consumidor (IPC), llevó a que se produjera una inestabilidad económica que fracturó el desarrollo de diferentes sectores, especialmente el industrial y el agrícola.

Finalmente, no se puede desconocer el impacto de la situación social y de seguridad del país en el desarrollo, el crecimiento y la competitividad de las empresas con participación de la mujer en Boyacá, pues «en la década del sesenta al problema del desempleo urbano se sumó a las tensiones sociales en el campo y a la aparición de los movimientos guerrilleros y populistas, que también tuvo consecuencias nefastas en el desarrollo económico del paísı (p. 8).

\subsection{Influencia de la condición de género en el desempeño de las mujeres como empresarias en Boyacá}

El estudio de la influencia de la condición de género en el desempeño de las mujeres en el entorno empresarial de Boyacá en las décadas de 1950, 1960 y 1970 está fundamentado en las entrevistas realizadas a seis mujeres empresarias que tuvieron trascendencia en la época, las cuales asumieron el rol en diferentes sectores -textil, alimentos, turismo y medicamentos - y a su vez fueron parte activa en el desarrollo y la prosperidad de unidades económicas que se consideraron insignia del desarrollo empresarial del departamento. Y tal como lo afirma Rebollo, Rodríguez y Jiménez (2016, p. 339), «en efecto, de espectadora pasiva, la mujer se transforma en autora material de su obra al tratar de construir una identidad pública vinculada asimismo a otra imagen social».

Los referentes obtenidos acerca de cómo funcionaban los negocios y el mundo empresarial boyacense de la época, evidencian que estos campos estaban dominados por los hombres. Los casos femeninos que hacían parte activa del empresariado eran aislados y se relacionaban con mujeres irreverentes poco tradicionales que no estaban de acuerdo con el rol femenino asignado por la sociedad, es decir, como se dice coloquialmente «echadas para adelante y de armas tomarı. Esas mujeres se salieron de los cánones tradicionalistas y tomaron decisiones que las llevaron poco a poco a incursionar en un entorno que estaba hecho para varones (Tabla 6). La mayoría de las mujeres que figuraron como empresarias no procedían de este departamento, sino que venían de otras jurisdicciones y hacían parte de familias que llegaron a esta región en busca de oportunidades laborales y comerciales. Este hallazgo ratifica lo establecido en el contexto cultural y conservador, pues evidencia que las mujeres pioneras del empresariado eran oriundas de departamentos cercanos a Boyacá en su mayoría, aunque su éxito y aceptación las llevó a crear arraigo y amor por estas tierras.

Yo llegué a Tunja sin conocer sino a mi hermano y mi hermano a mí; desde entonces ha sido cuarenta años de mi vida que voy a cumplir ahorita en junio que llegue a Tunja, entonces es toda una vida. Entonces fue como empezar de cero... le debo mucho a esta ciudad... y Boyacá nos ha dado todo y amamos Boyacá (entrevista con empresaria 2, 16 de abril de 2016 ). 
En lo que se refiere directamente al empresariado femenino, es importante resaltar que, sumado al coraje, carácter o valor de las mujeres empresarias, existía un antecedente de familia que las relacionaba de forma directa o indirecta con el mundo de los negocios, es decir, venían de linajes cuyo sustento estaba dado por actividades de intercambio comercial ejercido en diferentes sectores. De esta forma, las actividades comerciales escogidas estaban dadas por los legados, las capacidades o la formación previa, en otras palabras, incursionaron en sectores que de alguna forma conocían, o bien porque sus padres los habían trabajado, o bien porque estaban al tanto del oficio al haber sido formadas previamente en el colegio, hogar o empleos. Lo anterior está acorde con estudios recientes que sostienen que "dirigir una empresa en un sector con el que existen fuertes vínculos, tanto a nivel de formación como por experiencia previa, implica un aumento en la satisfacción personal y profesional" (Blasco, Brusca, Esteban y Labrador, 2016, p. 83). Esto permite inferir que desde mediados del siglo XX hasta las primeras décadas del siglo XXI se ha marcado una trayectoria donde hay una gran influencia del entorno familiar de negocios, que repercute en la proyección de mujeres emprendedoras, lo cual realmente aplica indistintamente a hombres y mujeres. La novedad del estudio está en determinar que el papel de la mujer abrió espacios en la década de 1960 en adelante también en el ámbito empresarial.

A pesar de su irreverencia y desacuerdo con el rol femenino de la época, como parte de su desarrollo personal y guiadas por su familia, las mujeres empresarias tenían que combinar el trabajo en sus negocios con el cuidado y la formación de sus hijos y esposos. Desde este punto de vista, se precisa un reto mayor, pues el solo hecho de pertenecer al género femenino les implicaba más sacrificio y esfuerzo para alcanzar su desarrollo profesional y el cumplimiento de sus metas, en comparación con el hombre que estaba dedicado cien por ciento a sus negocios. Desde entonces, y de acuerdo con Amaya (2014), la mujer colombiana como líder ha demostrado su capacidad para sobrellevar a la par responsabilidades, permitiéndole ser más proactiva en cuanto a sus conocimientos y actitud de liderazgo donde se da un equilibrio entre su vida laboral, familiar y personal (Tabla 6).

Mi padre no quiso dejarme ir a estudiar a la universidad a Bogotá, porque él decía estas palabras: "No la voy a dejar ir a Bogotá como perro sin amo. ¿A hacer qué?, y aquí en la casa tiene todo, no le hace falta nada, no hay necesidad"... Pero yo decía: "Yo no, yo no quiero, yo no quiero ser eso... Y yo toda la vida quise y luché por ser independiente (entrevista con empresaria 2, 16 de abril de 2016).

Así, aunque el escenario ofrecía desventaja para la mujer por los lineamientos sociales de la época, la condiciones de género para el desempeño de las mujeres que decidieron ser empresarias no fue una limitante sino un aliciente, pues la tenacidad de ellas hizo que esta condición de empresaria, madre y esposa se convirtiera en un reto o una oportunidad de mejora, que ayudado por la constancia y perseverancia han llevado a sus empresas al crecimiento y obtener un lugar en la historia empresarial del departamento, con una duración de más de cuarenta años en el mercado. Así, «la mujer empresaria está sobre todo motivada por una necesidad económica, aunada a la necesidad de contar con la flexibilidad para atender las responsabilidades familiares»» (Camarena y Saavedra, 2016, p. 24). 
Por otro lado, sobre el origen empresarial femenino, en la mayoría de los casos, las empresarias boyacenses iniciaron sus negocios gracias a herencias o capitales provenientes de sus padres o de sus esposos, pero sus inicios reales estuvieron marcados por hechos trascendentales que fueron base del comienzo de su aventura empresarial, es decir, antes de tener sus negocios fueron empleadas desde muy niñas, pues como un acto de rebeldía decidieron tomar iniciativas diferentes para no seguir el destino de ser estrictamente amas de casa como las demás. Por tal motivo, estas mujeres solo cuentan con una educación formal que no trasciende más allá del bachillerato; sin embargo, su tenacidad y ganas de superación las ha llevado a hacer cursos y aplicar a capacitaciones ofrecidas por entidades públicas y privadas, con el fin de mantener sus empresas y hacerles frente a sus competidores.

Cabe resaltar que el escenario y entorno inicial del empresariado femenino en Boyacá trascendió el empleo y desarrollo de la mujer de esa época, al preferir emplear a otras mujeres en sus unidades de negocio, ratificando la idea de fondo de visibilizar el trabajo femenino como una expresión de avanzada en el proceso de modernización de una sociedad tradicionalmente machista, vinculando más mujeres para que fueran parte activa del mundo laboral y empresarial combinando su roles socialmente instituidos en el hogar, lo cual aún en el siglo XXI sigue valorándose positivamente para el ámbito social, económico y cultural, tal como lo menciona Lomelí ( 2015, p. 28): «una mujer que se le permita contribuir al patrimonio familiar sin descuidar su hogar, dará un mayor rédito a la sociedad en la generación de empleos con horarios más flexibles y mejor pagadosı.

Pese a esto no se puede desconocer el hecho de que el incremento de espacios empresariales y laborales conquistados por las mujeres en los siglos XX y XXI, con mayor acento en la libertad económica que ella ha conquistado en el medio social, ha tenido efectos importantes en el cambio de roles entre lo femenino y lo masculino que impactan en el modelo de familia moderna y posmoderna. Esto se debe a que, durante décadas, alrededor del concepto que se ha construido socialmente sobre el trabajo se han presentado entre hombres y mujeres estereotipos, discursos y argumentos que reproducen la división sexual de trabajo que se apoya en la diferencia de los sexos que se traducen en desigualdades, a partir de las representaciones que les asignan el lugar que deben ocupar los hombres y las mujeres en la sociedad y en los ramos de actividades en los que pueden incursionar (Cataño, 2016).

Hoy en día tengo preferencia por emplear mujeres en esta droguería, como usted puede ver todas son mujeres, ellas tienen sus hijos, son mujeres cabeza de familia, eso me gusta, pues yo también luché mucho con mis hijos, a mí me tocó el tema fuerte de trabajar, tener a mis niños, y ser esposa. (entrevista con empresaria 4, 28 de enero de 2017)

De igual manera, la preferencia por el género femenino para el empleo está ligada al enfoque y cultura empresarial, es decir, en su labor como empresarias siempre estuvieron presentes aquellos principios y valores inculcados por su familia y en especial sus madres; valores relacionados con el respecto, la honradez, el cumplimiento, la rectitud, la honestidad, entre otras virtudes que les sirvieron, no solo para incursionar en el sector, sino también para crear o generar confianza y admiración ante otros empresarios masculinos que funcionaban como aliados comerciales, proveedores, clientes, etc. Además, las mujeres también 
sabían que «la innovación es un factor clave para la productividad, competitividad y crecimiento económico de organizaciones») (Ortiz, Morales y León, 2014, p. 161).

Desde otra perspectiva, la investigación también demostró que la condición de género sí tenía trascendencia desde el punto de vista legal y en la organización formal de las empresas de la época -1950-1979-. Es decir, aunque las mujeres empresarias eran las que mayor aporte hacían al desarrollo de sus negocios, en términos formales y legales los hombres eran los que las representaban -esposos o familiares-, esto se debía a que en algunos campos las grandes transacciones económicas solo eran funcionales entre hombres, pues, como ya se mencionó, el mundo empresarial en su mayoría estaba dominado por ellos y se manifestaba una cultura machista.

Con relación al contexto social y económico, se evidenciaba que estaba definido por estándares o criterios emanados de líderes masculinos que establecían un rol para la mujer que no iba más allá del hogar, por tanto, los fines y las metas de las mujeres se enmarcaban dentro del tejido familiar. A pesar del escenario inicial, las mujeres entrevistadas fueron agentes activos dentro de su actividad económica, ejercieron su papel de empresarias respetando y acatando las normas que regulan el mercado cumpliéndolas a cabalidad, fundamentadas en las bases éticas y los principios culturales positivos que recibieron en sus hogares, es decir, aunque no se hablaba en el momento de responsabilidad social, estas siempre propendieron a tomar decisiones que favorecieran el desarrollo no solo de su empresa, sino de la economía de la región. Además, demostraron capacidades y habilidades relacionadas con liderazgo, motivación, negociación, entre otras que son propicias y necesarias para surgir en el mundo de los negocios (Tabla 6).

Pero además de sus principios y arraigos culturales que favorecían su desarrollo empresarial, las empresarias asienten que para que una empresa surja, independiente del género, se requiere tenacidad, perseverancia, disciplina, cariño por lo que se hace, empeño, dedicación, trabajo arduo y visión, de tal forma que se pueda innovar y diferenciarse de la competencia.

Y yo creo que lo más necesario es el cariño que uno le ponga a su trabajo, puede ser a lo que sea, trabajo, el negocio, lo que sea, cualquier empresa sobresale es por eso, por el juicio, por el amor, por la dedicación, digamos, que uno diga dedicarle el tiempo que después voy a los frutos (entrevista con empresaria 1, 4 de marzo de 2016). 
Tabla 6. Relación entre la condición de género y su influencia en el desempeño como empresarias -1950-1979-

\begin{tabular}{|l|l|}
\hline \multicolumn{1}{|c|}{ Roles de género tradicional } & \multicolumn{1}{c|}{$\begin{array}{c}\text { Roles de género mujeres } \\
\text { empresarias }\end{array}$} \\
\hline $\begin{array}{l}\text { Papel secundario en el ámbito } \\
\text { empresarial: modelo de empresario } \\
\text { de éxito hombre de negocio. }\end{array}$ & $\begin{array}{l}\text { Propietarias y gerentes - crearon } \\
\text { empresa en su mayoría con ahorros } \\
\text { propios y algunas con apoyo de sus } \\
\text { familias-. }\end{array}$ \\
\hline $\begin{array}{l}\text { Las mujeres se dedicaban a } \\
\text { desempeñar el papel tradicional de } \\
\text { esposas, madres de familia y amas } \\
\text { de casa. }\end{array}$ & $\begin{array}{l}\text { Combinaban el trabajo en sus } \\
\text { negocios con el cuidado y formación } \\
\text { de sus hijos y la responsabilidad del } \\
\text { hogar. }\end{array}$ \\
\hline $\begin{array}{l}\text { Las mujeres sienten compromiso } \\
\text { con las normas predeterminadas } \\
\text { socialmente. }\end{array}$ & $\begin{array}{l}\text { Las mujeres son irreverentes } \\
\text { y se reúsan a seguir los roles } \\
\text { tradicionales. }\end{array}$ \\
\hline Enfoque conservador. & $\begin{array}{l}\text { La condición de género no fue } \\
\text { limitante sino un aliciente. Proyectos } \\
\text { compatibles con las necesidades de } \\
\text { su entorno familiar. }\end{array}$ \\
\hline
\end{tabular}

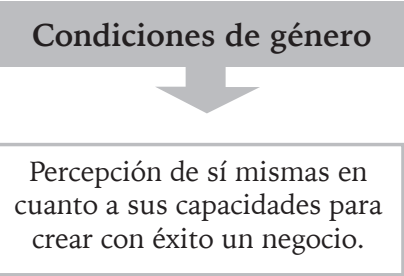

Flexibilización de los estereotipos de los roles de las mujeres.

Incursión de las mujeres en un nuevo rol.

Establecimiento de políticas empresariales que favorecen la empleabilidad de las mujeres

Fuente. Elaboración propia.

\section{Conclusiones}

L as mujeres tenían una participación accionaria promedio de $33 \%$, según figura en los papeles de constitución; sin embargo, a pesar de dicha participación, su rol era poco activo, pues el estudio muestra que de 158 empresas analizadas en tan solo $15 \%$ figuraba legalmente una mujer como directiva conjunta o representante legal del negocio. Esta situación, según análisis de otros autores e investigaciones previas, solo es el resultado de las condiciones culturales, económicas y políticas que caracterizaban el país y a Boyacá en ese momento.

Por otro lado, en lo que respecta a la actividad económica, la figura jurídica y la duración estimada de las empresas en las cuales las mujeres fueron partícipes fue evidente una mayor contribución de los sectores servicios y comercio. Estas activi- dades empresariales son congruentes con los conocimientos previos de las empresarias, ya que como madres y esposas estaban al tanto de las demandas y los requerimientos de las familias en cuanto a productos del hogar y necesidades de asistencia integral.

Desde el punto de vista legal, sobresalen significativamente las sociedades limitadas, con una participación promedio de $89 \%$ de la década de 1950 a finales de la década de 1970. La aceptación de esta figura jurídica tiene que ver con el hecho de que presentaba mayores facilidades en cuanto a constitución, responsabilidad, dirección y beneficios. Finalmente, se tiene que en el momento de la constitución, aproximadamente, los empresarios y las empresarias estimaban un periodo de duración de sus organizaciones de siete años. 
En cuanto al análisis de la incidencia del género en el desempeño de las empresarias, se puede concluir que el papel activo de la mujer en las décadas de 1950, 1960 y 1970 en los negocios de la región fue poco representativo, aun así, las mujeres que participaron no solo fueron pioneras y pusieron su marca en contra de las imposiciones sociales de la época, sino que demostraron que a pesar de las circunstancias y dificultades, su constancia, perseverancia, disciplina y valor les permitió abrirse paso en el sector empresarial y ser ejemplo para otras mujeres que hoy en día hacen parte de este entorno.

Los hechos positivos no impidieron reconocer que la condición de género en el desempeño de las mujeres como empresarias fue un factor relevante, en la medida en que presentaba limitaciones y barreras de entrada. En otras palabras, aunque el mundo de los negocios no estaba $100 \%$ blindado para las mujeres, la cultura, las creencias y el machismo lo hacían más difícil y poco atractivo para la mayoría de ellas.

La conducta de las empresarias que contribuyeron a forjar el desarrollo social y económico del departamento estuvo motivada por sus familias y los principios de estas, es decir, se adoptaron e imitaron conductas acordes con su desarrollo individual y colectivo cercano. Así, desarrollaron actitudes y sentimientos comunes que expresados públicamente les generaban confianza para transmitir creencias y tradiciones que podían integrarse al mundo de los negocios.

Por otro lado, las empresarias provenían de padres empleados o empresarios o dedicados a los negocios en el sector comercial y de servicios, así su nivel de vida y su posición heredada en el entorno empresarial, no solo les facilitaba la incursión en el medio, sino que además les dejaba unas bases en cuanto a apoyo económico se refiere.

Las mujeres empresarias aportaron al desarrollo y crecimiento económico de la región mediante la generación de empleos de calidad y la creación de unidades empresariales competitivas, además, han mantenido una mentalidad de innovación, constancia y proyección. De esta forma, están a la vanguardia en cuanto a servicios y productos, así mismo, tienen capacitación permanente para contar con los conocimientos administrativos y de mercados necesarios que les permiten ser competitivas y mantenerse vigentes en el mercado.

A partir de la investigación en referencia, sería procedente realizar estudios comparativos que permitan indagar y profundizar en aspectos básicos de las empresas, las empresarias y las prácticas administrativas, con el fin de conocer la contribución que han tenido otras regiones en relación con el crecimiento y desarrollo económico del país.

En cuanto a la línea de investigaciones futuras, sería muy importante indagar sobre cómo surgió el empresariado femenino en otras regiones de Colombia y en otros países latinoamericanos, para analizar de manera más amplia cómo el rol femenino se abrió campo en el mundo de los negocios como parte del proceso de modernización social, aportando un nuevo enfoque de análisis que complemente los estudios hechos desde los ámbitos educativos, políticos y culturales que hasta el momento se conocen. 


\section{Referencias}

Amaya, Á. (2014). Liderazgo de la mujer colombiana (Artículo de especialista, Universidad Militar Nueva Granada, Bogotá, Colombia).

Bernal, S., Cañas, A., Castillo, J., Cortés, S., Corredor, A. y Quintero, C. (2011). Auge minero-energético y estructura productiva en Colombia. Debates de Coyuntura Económica, 87. Recuperado de http:// www.repository.fedesarrollo.org.co/bitstream/ handle/11445/673/DCEyS_No_87.pdf?sequence $=1$

Blasco, P., Brusca, I., Esteban, L. y Labrador, M. (2016). La satisfacción de las mujeres empresarias: factores determinantes. Contabilidad y Negocios, 11(21), 68-92. Recuperado de http://www.redalyc. org/pdf/2816/281648512005.pdf

Boserup, E. (1970). Women and Economic Development. New York: St. Martin's Press.

Camarena, M. y Saavedra, M. (2016). Un estudio de las PYME lideradas por mujeres en Latinoamérica. Revista Universitaria Ruta, 18(1), 1-27. Recuperado de http://revistas.userena.cl/index.php/ruta/article/ view $/ 789 / 864$

Cataño, L. (2016). Mujeres emprendedoras en Colombia: una cuestión de género (Tesis de maestría, Universidad Federal do Paraná, Curitiba, Brasil).

Contreras, F., Pedraza, J. y Mejía, X. (2012). La mujer y el liderazgo empresarial. Diversitas, 8(1), 183-194. http://dx.doi.org/10.15332/s17949998.2012.0001.12

Dávila, C. (2012). Empresariado en Colombia: perspectiva histórica y regional. Bogotá: Universidad de los Andes.

Díaz, A. (1972). La economía colombiana y el comercio exterior en el período 1950-1970. Revista Coyuntura Económica, 2(3). Recuperado de http:// www.repository.fedesarrollo.org.co/bitstream/ handle/11445/2842/Co_Eco_Octubre_1972 Economia\%20Colombia\% 20 y $\% 20$ comercio $\% 2 \overline{0}$ exterior.pdf? sequence $=2$ \&isAllowed $=y$

Engels, F. (2000). El origen de la familia la propiedad privada y el estado. Bogotá: Géminis.
Garay, L. (1998). Colombia: estructura industrial e internacionalización 1967-1996. Recuperado de http://www.banrepcultural.org/blaavirtual/ economia/industrilatina/202.htm

Garbiras, M. (2010). El liderazgo de la mujer empresaria en Colombia: una mirada desde el saber, el poder y la sexualidad en las organizaciones privadas $y$ mix tas (Tesis de grado, Pontificia Universidad Javeriana, Bogotá, Colombia).

Hernández, I. (2005). Forma legal, innovación y productividad de las firmas en la industria manufacturera colombiana. Cuadernos de Economía, 24(42), 135-160. Recuperado de https://revistas. unal.edu.co/index.php/ceconomia/article/ view/9085

Kalmanovitz, S. y López, E. (s. f.). La agricultura en Colombia entre 1950 y 2000. Recuperado de http:// www.banrep.org/docum/ftp/borra255.pdf

Lamas, M. (2003). La perspectiva de género. Revista de Educación y Cultura de la sección 47 del SNTE. (8), 14-20. Recuperado de http://www.latarea.com. $\mathrm{mx} /$ articu/articu8/lamas8.htm

Lomelí García, G. (2015). El papel de la mujer como emprendedora y formador dentro de su hogar. México: Partido Acción Nacional.

Londoño, B. y Gómez, D. (2010). Diez años de investigación jurídica y sociojurídica en Colombia: balances desde la Red Sociojurídica (t. 2). Bogotá: Universidad de La Sabana.

Londoño, P. (1995). Las colombianas durante el siglo XIX. Recuperado de http://www.banrepcultural.org/ node/73270

Macho Morales, E. y Rosales Ortega, R. (2011). Empresarialidad femenina y redes sociales en San Pedro Tultepec de Quiroga, estado de México. Cuadernos de Geografía: Revista Colombiana de Geografía, 20(1), 85 - 101. Recuperado de https:// revistas.unal.edu.co/index.php/rcg/article/ view/23069/36711

Maldonado, A. (2010). La evolución del crecimiento industrial y transformación productiva en Colombia 1970-2005: patrones y determinantes. Bogotá: Universidad Nacional de Colombia. 
Moghadam, V. (1999). Gender and globalization: female labor and women's mobilization. Journal or world- sistems research. 5(2), https://doi. org/10.5195/jwsr.1999.139

Oficina Internacional del Trabajo Ginebra. (2007). La promoción de empresas sostenibles. Recuperado de http://www.ilo.org/public/spanish/standards/ relm/ilc/ilc96/pdf/rep-vi.pdf

Ortiz, C., Morales, M. y León, E. (2014). Trayectorias y condiciones para la innovación en empresas de propiedad femenina: análisis comparativo de dos ciudades colombianas. Revista Facultad de Ciencias Económicas: Investigación y Reflexión, 22(2), 159-184. Recuperado de http://www.scielo.org. $\mathrm{co} /$ scielo.php? script $=$ sci_abstract\&pid $=$ S0121$68052014000200010 \& \operatorname{lng}=$ es $\& n r m=$ is.

Rebollo, M., Rodríguez, R. y Jiménez, R. (2016). Gestión y liderazgo empresarial con perspectiva de género: voces y experiencias. Cuestiones de género: de la igualdad y la diferencia, 11, 329-350. Doi: 10.18002/cg.v0i11.3186

Reyes C. (2015). Cambios en la vida femenina durante la primera mitad del siglo XX. Credencial Historia, 68. Recuperado de http://www.banrepcultural. org/blaavirtual/revistas/credencial/agosto95/ agosto3.htm

Rodríguez, D., Jiménez, C. y Rebollo, C. (2016). Gestión y liderazgo empresarial con perspectiva de género: voces y experiencias. Cuestiones de género: de la igualdad y la diferencia. (11), 329-350

Segura, M. y Pintor, L. (2010). Una visión desde el empresarismo femenino en Colombia y su potencial de internacionalización. Recuperado de http://www. konradlorenz.edu.co/images/publicaciones/ suma_negocios_working_papers/2011-v1-n1/01empresarismo-femenino.pdf

Semana (9 octubre 1995). Las 50 mujeres más importantes de Colombia. Recuperado de http://www.semana. com/especiales/articulo/las-50-mujeres-masimportantes-de-colombia/26739-3

Serret, E. (2008). ¿Qué es y para qué es la perspectiva de género? Recuperado de http://equidad.pueg.unam. $\mathrm{mx} /$ sites/default/files/files/oax09.pdf

Zabludovsky, G. (2015). Las mujeres en los ámbitos de poder económico y político de México. Revista Mexicana de Ciencias Políticas y Sociales, 60(223), 6194. Recuperado http://www.revistas.unam.mx/ index.php/rmcpys/article/view/45380 